Faculté des sciences sociales École de psychologie

\title{
LES ATTITUDES ET LA PRÉSENCE DANS L'EXPÉRIENCE DE LA RELATION D'AIDE PAR VIDÉOCONFÉRENCE
}

Andréanne SIMARD ${ }^{1,2, *}$, Alexane BARIBEAU-LAMBERT ${ }^{1}$, Andrée-Anne BEAUDOIN-JULIEN ${ }^{1}$, Frédéric GRONDIN ${ }^{1,2,3}$ et Philip JACKSON $1,2,3$

'École de psychologie, Université Laval, Québec, Canada

${ }^{2}$ Centre de recherche CERVO, Québec, Canada

${ }^{3}$ Centre interdisciplinaire de recherche en réadaptation et intégration sociale (CIRRIS), Québec, Canada

*andreanne.simard.4@ulaval.ca

\section{Pour citer l'article}

Simard, A., Baribeau-Lambert, A., Beaudoin-Julien, A.-A., Grondin, F., \& Jackson, P. (2018). Les attitudes et la présence dans l'expérience de la relation d'aide par vidéoconférence. Psycause : Revue scientifique étudiante de l'École de psychologie de l'Université Laval, 8(2), 18-20. 
niveau d'AP demeure sous les recommandations nationales, renforçant l'importance de promouvoir davantage I'AP pendant et après les traitements. Finalement, puisqu'il s'agit de la première étude cherchant à dresser le portrait longitudinal de I'AP mesurée subjectivement et objectivement, ces résultats devront être répliqués auprès d'échantillons plus grands et de patients ayant différents types de cancer.

\section{Références}

Brunet, J., Amireault, S., Chaiton, M., \& Sabiston, C. M. (2014). Identification and prediction of physical activity trajectories in women treated for breast cancer. Annals of epidemiology, 24, 837-842. doi:10.1016/j. annepidem.2014.07.004

Harrison, S., Hayes, S. C., \& Newman, B. (2009). Level of physical activity and characteristics associated with change following breast cancer diagnosis and treatment. PsychoOncology, 18, 387-394. doi:10.1002/pon.1504

Oberski, D. (2016). Mixture models: Latent profile and latent class analysis. In J. Robertson, \& M. Kaptein (Eds.), Modern statistical methods for $\mathrm{HCl}$ (pp. 275-287). Basel, Suisse: Springer.
Schmitz, K. H., Courneya, K. S., Matthews, C., Demark-Wahnefried, W., Galvão, D. A., Pinto, B. M., ... Schwartz, A. L. (2010). American college of sports medicine roundtable on exercise guidelines for cancer survivors. Medicine and Science in Sports and Exercise, 42, 1409-1426. doi:10.1249/MSS.0b013e3181e0c112

Société canadienne du cancer (2017). Statistiques sur le cancer du sein. Récupéré sur le site de la Société canadienne du cancer le 10 novembre 2017 : www.cancer.ca

Speck, R. M., Courneya, K. S., Mâsse, L. C., Duval, S., \& Schmitz, K. H. (2010). An update of controlled physical activity trials in cancer survivors: A systematic review and meta-analysis. Journal of Cancer Survivorship, 4, 87-100. doi:10.1007/ s11764-009-0110-5

\title{
LES ATTITUDES ET LA PRÉSENCE DANS L'EXPÉRIENCE DE LA RELATION D'AIDE PAR VIDÉOCONFÉRENCE
}

\author{
Andréanne SIMARD ${ }^{1,2}$, Alexane BARIBEAU-LAMBERT ${ }^{1}$, Andrée-Anne BEAUDOIN-JULIEN ${ }^{1}$, \\ Frédéric GRONDIN ${ }^{1,2,3}$ et Philip JACKSON $1,2,3$ \\ ${ }^{1}$ École de psychologie, Université Laval, Québec, Canada ${ }^{2}$ Centre de recherche CERVO, Québec, Canada ${ }^{3}$ Centre \\ interdisciplinaire de recherche en réadaptation et intégration sociale (CIRRIS), Québec, Canada
}

\section{Introduction}

L'utilisation des nouvelles technologies de la communication est en croissance dans plusieurs domaines, dont celui de la psychothérapie (Lombard, Ditton \& Weinstein, 2009). Malgré les résultats des études sur la téléthérapie (thérapie par vidéoconférence) rapportant une efficacité équivalente à la thérapie en face-àface (Simpson \& Reid, 2014), cette technique d'intervention reste sous-utilisée au Canada (Simms, Gibson \& O'Donnell, 2011).

Afin d'augmenter I'utilisation et la qualité des interactions en téléthérapie, deux facteurs centraux au développement de la relation 
thérapeutique seront étudiés, soit les attitudes qu'entretiennent le client et le thérapeute envers la téléthérapie et l'expérience de présence vécue pendant les séances (Bouchard, Dumoulin, Michaud \& Gougeon, 2011). La présence réfère à l'impression d'être plongé dans l'interaction avec une autre personne dans un même environnement (Bouchard, Robillard, Marchand, Renaud \& Giuseppe, 2007). Puisque des attitudes négatives envers la téléthérapie peuvent freiner son utilisation et que la présence fait partie intégrante d'une expérience thérapeutique positive, ce projet a pour objectif de mesurer les attitudes et la présence en contexte de relation d'aide par vidéoconférence comparativement à un contexte face-à-face, ainsi que la force de l'association entre ces deux concepts.

Conformément aux écrits qui mentionnent que plusieurs réserves sont émises envers la téléthérapie (p. ex., problèmes techniques, crainte de contact impersonnel; Barak, Hen, Boniel-Nissim \& Shapira, 2008), il est attendu que: (a) les attitudes des participant(e)s soient moins favorables envers la thérapie en vidéoconférence qu'envers la thérapie en face-àface; (b) le niveau de présence soit inférieur en vidéoconférence, conformément aux écrits de Bouchard et collaborateurs (2011); et que (c) les attitudes et la présence présentent une corrélation significative positive, comme le soulignent Jerome et Jordan (2007).

\section{Méthode}

L'échantillon est composé de 29 étudiantes et étudiants universitaire (26 femmes), âgé(e)s en moyenne de 25,45 ans (É- $T=5,38$ ), formé(e)s et ayant de l'expérience (minimum de trois mois) en relation d'aide. Les participant(e)s utilisent fréquemment l'ordinateur $(M=$ 6,72 jours/semaine), mais très peu la vidéoconférence $(65,5 \%$ ne l'utilisent pas).
Des dyades de relation d'aide sont créées en attribuant aléatoirement le rôle de "thérapeute» ou de «client(e)» à chaque participant(e). Les participant(e)s réalisent deux conditions, avec le même rôle, l'une en face-à-face, l'autre en vidéoconférence, à une semaine d'intervalle (ordre contrebalancé). Les participant(e)s remplissent les questionnaires d'attitudes envers les deux types de thérapie, puis entament la séance de relation d'aide de 20 minutes à propos du sujet choisi par le/la «client(e)». Ils remplissent ensuite le questionnaire de présence.

\section{Résultats et discussion}

Premièrement, les résultats montrent que les attitudes négatives des participant(e)s sont significativement plus élevées envers la téléthérapie $(M=12,172 ; \dot{E}-T=3,674)$ qu'envers la thérapie en face-à-face $(M=8,448 ; \dot{E}-T=$ 2,983), $t(28)=5,348 ; p<0,001 ; d=0,998$, confirmant certaines études précédentes (p. ex., Simms et coll., 2011). Cependant, les participant(e)s de l'étude passent en moyenne un nombre d'heures important par semaine sur l'ordinateur, ce qui suggère que les attitudes négatives envers la téléthérapie persistent bien que les participant(e)s utilisent régulièrement l'ordinateur. II se pourrait que ces attitudes négatives soient plutôt expliquées par la faible utilisation de la vidéoconférence au sein de l'échantillon.

Deuxièmement, les résultats indiquent que la présence est plus élevée lors des séances en face-à-face $(M=91,058 ; \dot{E}-T=4,851)$ que lors des séances en vidéoconférence $(M=$ $70,144 ; \dot{E}-T=16,210), t(25)=6,487 ; p<0,001$; $d=1,274^{4}$. II serait intéressant de reproduire cette étude en mesurant objectivement les variables de la qualité du son, de l'image et de la connexion Internet, afin de vérifier si ces éléments influencent la présence ressentie par les participant(e)s. La présence étant centrale

4 Deux participant(e)s ont été retirés des analyses et un(e) participant(e) n'a pas complété l'une des deux conditions, expliquant ainsi la différence par rapport aux degrés de liberté. 
dans l'expérience thérapeutique (Bouchard et coll., 2011), des stratégies visant à mieux la comprendre, puis à la maximiser sont primordiales pour l'avenir de la téléthérapie.

Troisièmement, les résultats montrent une association négative forte entre la présence et les attitudes négatives $\left(r=-0,557 ; r^{2}=0,333\right.$; $p<0,001$ ). Autrement dit, moins les attitudes envers une modalité de thérapie sont favorables, moins la présence rapportée dans l'interaction est élevée, ce qui supporte l'hypothèse. L'étude de cette relation n'a pas de précédent dans les écrits, ces résultats devront ainsi être reproduits. Si une relation de prédiction est découverte, il serait possible d'intervenir pour améliorer les attitudes envers la téléthérapie afin d'augmenter la présence vécue en séance.

Dans l'ensemble, les résultats montrent donc que les attitudes négatives envers la téléthérapie persistent malgré une utilisation élevée de l'ordinateur et que la présence, moins élevée en téléthérapie, serait un élément pertinent à cibler pour améliorer la qualité de la téléthérapie. Bien que ce projet soit novateur et qu'il possède un devis rigoureux, il est toutefois important de considérer ses limites, soit le caractère homogène de l'échantillon (domaine d'étude, âge, sexe féminin) et le devis expérimental ne représentant pas un réel contexte de psychothérapie.

\section{Références}

Barak, A., Hen, L., Boniel-Nissim, M., \& Shapira, N. (2008). A comprehensive review and a meta-analysis of the effectiveness of Internetbased psychotherapeutic interventions. Journal of Technology in Human Services, 26, 109160. doi:10.1080/15228830802094429

Bouchard, S., Dumoulin, S., Michaud, M., \& Gougeon, V. (2011). Telepresence experienced in videoconference varies according to emotions involved in videoconference sessions. Dans B. K. Wierderhold, S. Bouchard, \& G. Riva (Eds.), Annual Review of CyberTherapy and Telemedicine (Vol. 9, pp. 104-107). San Diego, CA: Interactive Media Institute.

Bouchard, S., Robillard, G., Marchand,A., Renaud, P., \& Giuseppe, R. (2007). Presence and the bond between patients and their psychotherapists in the cognitive-behavior therapy of panic disorder with agoraphobia delivered in videoconference. Presence, (October), 89-95.

Jerome, L. W., \& Jordan, P. J. (2007). Psychophysiological perspective on presence: The implications of mediated environments on relationships, behavioral health and social construction. Psychological Services, 4, 75-84. doi:10.1037/1541-1559.4.2.75

Lombard, M., Ditton, T. B., \& Weinstein, L. (2009). Measuring presence: The Temple Presence Inventory. Proceeding of Presence 2009: The $12^{\text {th }}$ International Workshop on Presence, 1-14.

Simms, D. C., Gibson, K., \& O'Donnell, S. (2011). To use or not to use: Clinicians' perceptions of telemental health. Canadian Psychology/ Psychologie Canadienne, 52, 41-51. doi:10.1037/a0022275

Simpson, S., \& Reid, C. L. (2014). Therapeutic alliance in videoconferencing psychotherapy: A review. Australian Journal of Rural Health, 22, 280-299. doi:10.1111/ajr.12149 